\title{
MAGNETIC FIELD DEPENDENCE OF PHONON-INDUCED DRAG CURRENT OF 2D CARRIERS
}

\author{
D. LEHMANN \\ Institut für Theoretische Physik, Technische Universität Dresden \\ 01062 Dresden, Germany \\ Cz. Jasiukiewicz and T. Paszkiewicz \\ Institute of Theoretical Physics, University of Wrocław \\ Pl. Borna 9, 50-205 Wroclaw, Poland
}

The magnetic field dependence of the drag current induced by beams of acoustic phonons is studied in a $2 \mathrm{D}$ gas of carriers, formed in a heterostructure. This drag current is related to the velocity of the center of mass of the gas. The elimination of the degrees of freedom related to the relative motion of the carriers leads to a Langevin equation for the center-of-mass position vector. From this equation one can obtain an expression for the induced current density which depends on carrier density-density correlation functions. An explicit formula for the time-integrated current density is derived taking into account all intra and inter Landau level transitions. Corresponding numerical results are in good agreement with the experimental patterns.

PACS numbers: $63.20 . \mathrm{Kr}, 73.40 .-\mathrm{c}$

\section{Introduction}

It is now well established that acoustic phonons can be used as a powerful probe of two-dimensional carrier gases (2DCG). Phonon drag imaging is a particularly useful technique because it gives information regarding the carrier phonon coupling as a function of both the phonon wave vector direction and its magnitude. To understand the images it is necessary to develop detailed theoretical models of the coupling including the effects of acoustic anisotropy. In this paper we extend our previous work [1] on the phonon drag of 2D holes in GaAs heterostructures to include the situation where a magnetic field is applied normal to the 2DCG. In quantising fields carriers are localised on orbits with radii much smaller than the average distance between defects [1]. Therefore, the drag current induced by phonons is related only to the motion of the center of mass (CM) of the carriers. The response of the 2DCG due to the phonon pulse, here the drag current $J(t)$, is proportional to the velocity of the CM. 


\section{The Langevin equation for the center of mass}

To describe the motion of CM we consider a (quasi-) 2DCG gas, containing $N$ carriers with effective mass $m^{*}$, interacting with phonons and impurities in the presence of a perpendicular quantising magnetic field. For simplicity, we assume that from the quantum subbands due to the spatial confinement of the carriers only the lowest one is occupied. The Hamiltonian consists of three terms of different nature

$$
\begin{aligned}
& H=\frac{1}{2 N m^{*}}[\boldsymbol{P}-N e \boldsymbol{A}(\boldsymbol{R})]^{2}+V\left(R_{z}\right) \\
& +\sum_{n, k} \varepsilon_{n} c_{n k}^{+} c_{n k}+\frac{1}{2} \sum_{q_{\|}} v\left(q_{\|}\right)\left(g\left(q_{\|}\right) \rho_{-} q_{\|} \rho q_{\|}-\hat{N}\right)+\sum_{Q} \hbar \omega_{Q} b_{Q}^{+} b_{Q} \\
& +\sum_{Q} h(Q) \mathrm{e}^{\mathrm{i} q \boldsymbol{R}}\left(b_{Q}+b_{-Q}^{+}\right) G\left(q_{z}\right) \rho_{-} q_{\|}+\sum_{q} u(q) \mathrm{e}^{\mathrm{i} \boldsymbol{q} \boldsymbol{R}} G\left(q_{z}\right) \rho_{-} \boldsymbol{q}_{\|} .
\end{aligned}
$$

The terms in the first line depend only on CM variables. $\boldsymbol{P}$ and $\boldsymbol{R}$ are the center-of-mass momentum and coordinate, respectively, $V\left(R_{z}\right)$ is the confinement potential of the carriers, the $z$-direction is perpendicular to the 2DCG, $A(R)$ is the vector potential, which we take in Landau gauge. The terms in the second line represent the motion of the carriers and of the phonons relative to the $\mathrm{CM}$ including carrier-carrier Coulomb interaction. The operators $c_{n k}^{+}$and $c_{n k}$ are related to the relative motion and create or annihilate a "relative" electron (hole) in the Landau level $n$ with the wave number $k, \varepsilon_{n}$ is the Landau level energy, $\rho_{q_{\|}}$ is the (in-plane) 2D Fourier transform of the carrier density operator and $v\left(q_{\|}\right)$is the 2D Fourier transform of the Coulomb potential. The functions $g\left(q_{\|}\right)$and $G\left(q_{z}\right)$ arise from the finite extension of the carrier wave functions along the $z$-axis and depend on the chosen confinement potential. The operators $b_{Q}^{+}$and $b_{Q}$ create and annihilate a phonon $Q$ in the substrate of the density $\rho$ and volume $V$. The index $Q$ stands for the wave vector $q$ and the polarization $\lambda, \omega_{Q}$ is the frequency of a phonon with the polarization vector $e(Q)$. The only coupling between the center of mass and the "relative" carriers (third line) is due to the carrier-impurity (c-im) and carrier-phonon (c-ph) interaction. The function $u(q)$ is the Fourier transform of the screened c-im potential and $h(Q)$ is the familiar c-ph matrix element, which includes both deformation potential and piezoelectric coupling

$$
\begin{aligned}
h(Q) & =\sqrt{\frac{\hbar}{2 V \rho \omega_{Q}}}\left\{\mathrm{i} \Xi_{\mathrm{D}} q e(Q)\right. \\
& \left.+2 e h_{14} \frac{e_{x}(Q) q_{y} q_{z}+e_{z}(Q) q_{x} q_{y}+e_{y}(Q) q_{z} q_{x}}{q^{2}}\right\},
\end{aligned}
$$

$\Xi_{\mathrm{D}}, h_{14}$ are the suitable coupling constants.

The motions of the center of mass and of the "relative" carriers have quite different natures. CM moves erratically as a classical "Brownian" particle with enormous mass $M=N m^{*}$, its motion is governed by a classical stochastic equation of the Langevin type. The motion of a huge number of interacting "relative" carriers is described by a statistical density matrix. 
The equation of motion for the center of mass follows from Eq. (1)

$$
\begin{aligned}
& M\left(\ddot{\boldsymbol{R}} \pm \omega_{\mathrm{c}} \dot{\boldsymbol{R}} \times \widehat{\boldsymbol{B}}\right) \\
& =\sum_{Q} q \operatorname{Im}\left\{\mathrm{e}^{\mathrm{i} q R_{(t)}} G\left(q_{z}\right)\left[2 h(Q)\left\langle b_{Q} \rho_{-} q_{\|}\right\rangle+u(q)\left(\rho_{-} q_{\|}\right\rangle\right]\right\},
\end{aligned}
$$

where the upper (lower) sign corresponds to electrons (holes). The terms on the right-hand side of Eq. (2) are the forces exerted on the center of mass due to the c-ph and c-im interaction, respectively. Using a procedure of $\mathrm{Hu}$ and $\mathrm{O}^{\prime} \mathrm{Connell}$ [2] to express the terms $\left\langle b_{Q} \rho_{-} q_{\|}\right\rangle$and $\left\langle\rho_{-} q_{\|}\right\rangle$(which are coupled to $R(t)$ ) by densitydensity correlations functions and neglecting all quadratic or higher order terms in $q \dot{\boldsymbol{R}}$ we can recast Eq. (2) into a quantum Langevin equation from which one may separate the drag forces $\boldsymbol{F}$ and the frictional forces $(\sim \dot{\boldsymbol{n}})$. Thus the right hand side of Eq. (2) can be written in the form

$$
\left\langle F^{(\mathrm{ph}, \mathrm{im})}(t)\right\rangle-\int_{-\infty}^{t}\left\langle M^{(\mathrm{ph}, \mathrm{im})}\left(t-t^{\prime}\right)\right\rangle \dot{\boldsymbol{R}}\left(t^{\prime}\right) \mathrm{d} t^{\prime} .
$$

The force $\left\langle F^{(\mathrm{im})}\right\rangle$ averaged over all impurity positions vanishes, so only phonons contribute to the drag force. Finally, we get for the time integrated drag current

$$
\int_{-\infty}^{\infty} \mathrm{d} t \boldsymbol{J}(t)= \pm \frac{\mu}{1+(\mu B)^{2}}\left[\left\langle\mathcal{F}^{(\mathrm{ph})}(0)\right\rangle \pm \mu\left(\left\langle\mathcal{F}^{(\mathrm{ph})}(0)\right\rangle \times B\right)\right],
$$

where $\mu=e N /\left(\left\langle\mathcal{M}^{(\mathrm{ph})}(0)\right\rangle+\left\langle\mathcal{M}^{(\mathrm{im})}(0)\right\rangle\right)$ is the mobility and $\langle\mathcal{F}(\omega)\rangle,\langle\mathcal{M}(\omega)\rangle$ are the Fourier transforms of $\langle\boldsymbol{F}(t)\rangle$ and $\langle M(t)\rangle$, respectively. We calculated the drag force $\left\langle\mathcal{F}^{(\mathrm{ph})}(0)\right\rangle$ in random phase approximation $(\mathrm{RPA})$ and neglected all higher order phonon processes and all processes involving simultaneously phonons and impurities

$$
\begin{aligned}
& \left\langle\mathcal{F}^{(\mathrm{ph})}(0)\right\rangle \\
& \quad=2 \sum_{Q} q \mathcal{N}_{Q}(0)|h(Q)|^{2} \operatorname{Im}\left[\frac{\left|G\left(q_{z}\right)\right|^{2} \chi^{(0)}\left(q_{\|},-\omega_{Q}\right)}{1-v\left(q_{\|}\right) g\left(q_{\|}\right) \chi^{(0)}\left(q_{\|},-\omega_{Q}\right)}\right] .
\end{aligned}
$$

$\mathcal{N}_{Q}(\omega)$ is the Fourier transform of the solution of the kinetic equation for the phonon distribution function [1] and $\chi^{(0)}\left(q_{\|}, \omega_{Q}\right)$ is the multi-subband polarizability for a 2DCG in the presence of a perpendicular magnetic field taking into account all the intra and inter Landau level transitions

$$
\chi^{(0)}\left(q_{\|}, \omega\right)=\sum_{n, n^{\prime}} \frac{C_{n n^{\prime}}\left(q_{\|} l_{\mathrm{B}}\right)\left(f_{n^{\prime}}-f_{n}\right)}{\varepsilon_{n^{\prime}}-\varepsilon_{n}-\hbar \omega-\mathrm{i} \delta},
$$

where $f_{n}$ is the Fermi equilibrium distribution function for the carriers and the function $C_{n n^{\prime}}(x)$ is related to the associated Laguerre functions $L_{n}^{m}(y)$ (cf. [2])

$$
C_{n n^{\prime}}(x)=\frac{1}{2 \pi l_{\mathrm{B}}} \frac{n !}{n^{\prime} !} \frac{x^{2\left(n-n^{\prime}\right)}}{2^{\left(n-n^{\prime}\right)}} \mathrm{e}^{-x^{2} / 2}\left[L_{n}^{\left(n-n^{\prime}\right)}\left(x^{2} / 2\right)\right]^{2} .
$$




\section{Results and conclusions}

In Fig. 1 the calculated images of the drag current for a (quasi) 2-dimensional hole gas at $B=0,0.25$, and $4.71 \mathrm{~T}$ are displayed. The important result is the apparent anticlockwise "rotation" of the axis about which the drag signal reverses polarity. This effect has been observed in experiments of Lehmann et al. [3]. To see
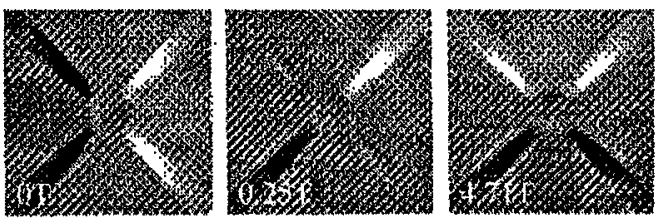

Fig. 1. The calculated images of the drag current in (quasi) $2 \mathrm{D}$ hole gas for various strengths of magnetic field.

how this comes about one must remember that according to Eq. (4) the pattern obtained in a field is the superposition of two components: the parallel one is due to the phonon momentum along the line joining the contacts and the transverse one is related to the phonon momentum perpendicular to this line. Their ratio is given by the factor $\mu B$. In the mentioned experiments $\mu B \approx 1$ for $B=0.25 \mathrm{~T}$. At $B=0$ only the parallel component contributes. At $B=4.71 \mathrm{~T}$ (i.e. $\mu B \gg 1$ ) the transverse component dominates giving the rotation of $90^{\circ}$. Strong evidence of this is seen in the experimental patterns for $4.71 \mathrm{~T}$, however, it is severely affected by noise. This is because the drag signal is proportional to the pre-factor $\left[1+(\mu B)^{2}\right]^{-1}$. The pattern for $B=0.25 \mathrm{~T}$ is the superposition of patterns for $B=0$ and for $\mu B \gg 1$.

We would like to thank EC for supporting this work under contract Nos. CHRX-CT94-0548 and CIPA CT93-0070.

\section{References}

[1] W.M. Gancza, Cz. Jasiukiewicz, A.J. Kent, D. Lehmann, T. Paszkiewicz, K.R. Strickland, R.E. Strickland, Semicond. Sci. Technol. 11, 1030 (1996).

[2] G.Y. Hu, R.F. O'Connell, Physica B 151, 33 (1988).

[3] D. Lehmann, A.J. Kent, K.R. Strickland, R.E. Strickland, M. Henini, Cz. Jasiukiewicz, T. Paszkiewicz, in: High Magnetic Fields in the Physics of Semiconductors, Eds. G. Landwehr, W. Ossau, Vol. 1, World Scientific, Singapore 1997, p. 345 . 